\title{
The effects of baicalin on piglets challenged with Glaesserella parasuis
}

\author{
Shulin Fu $u^{1,2+}$, Ronghua Yin ${ }^{1,2+}$, Sanling Zuo ${ }^{1,2+}$, Jun Liu ${ }^{1,2}$, Yunfei Zhang ${ }^{1,2}$, Ling Guo ${ }^{1,2}$, Yinsheng Qiu ${ }^{1,2^{*}} \mathbb{D}_{\text {, }}$ \\ Chun Ye $\mathrm{Y}^{1,2}, \mathrm{Yu} \mathrm{Liu}^{1,2}$, Zhongyuan $\mathrm{Wu}^{1,2}$, Yongqing Hou ${ }^{1,2}$ and Chien-An Andy Hu $\mathrm{H}^{1,3}$
}

\begin{abstract}
Glaesserella parasuis (G. parasuis) causes porcine vascular inflammation and damage. Baicalin is reported to have antioxidant and anti-inflammatory functions. However, whether baicalin protects piglets against G. parasuis challenge and the potential protective mechanism have not been investigated. Therefore, in this study, we comprehensively examined the protective efficacy of baicalin in piglets challenged with G. parasuis and the possible protective mechanism. Our results show that baicalin attenuated the release of the inflammation-related cytokines interleukin (IL) $1 \beta$, IL6, IL8, IL10, and tumour necrosis factor a (TNF-a) and reduced high mobility group box 1 (HMGB1) production and cell apoptosis in piglets infected with G. parasuis. Baicalin also inhibited the activation of the mitogen-activated protein kinase (MAPK) signalling pathway and protected piglets against G. parasuis challenge. Taken together, our data suggest that baicalin could protect piglets from G. parasuis by reducing HMGB1 release, attenuating cell apoptosis, and inhibiting MAPK signalling activation, thereby alleviating the inflammatory response induced by the bacteria. Our results suggest that baicalin has utility as a novel therapeutic drug to control G. parasuis infection.
\end{abstract}

Keywords: baicalin, Glaesserella parasuis, piglets, inflammatory response, protection

\section{Introduction}

Glaesserella parasuis (G. parasuis), which belongs to the family Pasteurellaceae, is responsible for Glässer's disease in pigs. The typical characteristics of Glässer's disease are fibrinous polyserositis, arthritis, meningitis, and frequent symptoms of pneumonia [1]. To date, 15 serovars have been identified with the heat-stable antigen extraction method [2], but a significant percentage of isolates has not been classified by this method [3]. Serovar 5 is thought to be highly virulent, and epidemiological studies have shown that serovars 4,5 , and 13 are the current epidemic strains in China [3]. With changes in the breeding patterns in pigs, the economic losses caused by G. parasuis have become more severe. Because there are

\footnotetext{
*Correspondence: qiuyinsheng6405@aliyun.com

†Shulin Fu, Ronghua Yin and Sanling Zuo contributed equally to this work

${ }^{1}$ Hubei Key Laboratory of Animal Nutrition and Feed Science, Wuhan

Polytechnic University, Wuhan 430023, People's Republic of China

Full list of author information is available at the end of the article
}

numerous serovars of G. parasuis that do not induce protective cross-immunity, the prevention and control of $G$. parasuis infection have become urgent problems [4].

The pathogenic mechanism of G. parasuis is currently unclear, so it is difficult to control infection. However, several virulence-related factors have recently been reported to be involved in its pathogenicity $[5,6]$. G. parasuis induces autophagy in the porcine alveolar macrophage (PAM) cell line 3D4/21 via the mitogen-activated protein kinase (MAPK) signalling pathway [7]. Additionally, deletion of the $r f a D$ and $r f a F$ genes involved in lipooligosaccharide (LOS) biosynthesis of $G$. parasuis decreases the secretion of proinflammatory cytokines in PAMs through regulation of the nuclear factor $\mathrm{\kappa B}(\mathrm{NF}-\mathrm{\kappa B})$ and MAPK signalling pathways during the infection process [8]. Previous research has shown that the AI-2/luxS quorum sensing system affects the growth and virulence of G. parasuis [9]. The bacterium also disrupts adherens junctions and initiates the epithelial-mesenchymal 
transition, leading to fibrinous polyserositis, which depends on the regulation of the canonical $W N T / \beta-$ catenin signalling pathway [10]. QseC-mediated osmotic stress resistance and biofilm formation regulate the density of G. parasuis [11]. These virulencerelated factors not only are important pathogenic factors but also elicit the host immune response [12] and are therefore considered as important drug targets for the prevention of Glässer's disease.

With the extensive use of antibiotics on pig farms, the phenomenon of bacterial resistance has become more serious. Therefore, screening for environmentally friendly efficacious drugs for which resistance has not been developed has become an urgent focus of disease control. Baicalin, extracted from Scutellariae Radix, has been shown to have potential therapeutic functions. Previous research has demonstrated that baicalin relieved the inflammation in L-02 and THLE2 liver cells induced by lipopolysaccharide (LPS) by upregulating taurine upregulated 1 (TUG1) [13]. Baicalin attenuated the intestinal disruption caused by challenge with Salmonella pullorum and modulated the gut microbiota in laying hens [14]. It also alleviated the inflammatory immune responses of chicken type II pneumocytes stimulated with avian pathogenic Escherichia coli (APEC) and may inhibit APEC biofilm formation and the expression of APEC virulence genes [15]. Baicalin also improved the health of mice and prevented their infection with Helicobacter pylori in a model of inflammation by interfering with the growth and virulence of $H$. pylori [16]. Baicalin protected mice against $S$. Typhimurium challenge by modulating both the bacterium's virulence and the host's immune response [17]. In our previous work, we found that baicalin could suppress the NF- $k B$ and NLRP3 inflammasome signalling pathways induced by G. parasuis in porcine aortic vascular endothelial cells (PAVECs) [18] and piglet mononuclear phagocytes (PMNPs) [19]. Baicalin reduced apoptosis triggered by $G$. parasuis via RAGE, MAPK, and AP-1 in PAVECs [20]. Baicalin also inhibited PKC-MAPK signalling pathway activation [21] and attenuated high-mobility group box 1 (HMGB1) secretion [22] in PMNPs stimulated by G. parasuis. In addition, baicalin could modulate long non-coding RNA and mRNA expression in PAVECs infected by G. parasuis [23]. However, whether baicalin can protect piglets against G. parasuis challenge has not been investigated.

In this study, we investigated the effects of baicalin in piglets challenged with G. parasuis. Our results suggest that baicalin has utility as a novel therapeutic drug to control G. parasuis infection in pigs.

\section{Materials and methods}

\section{Bacterial strain and growth conditions}

Glaesserella parasuis strain SH0165 serovar 5 was isolated from the lung of a commercially produced pig with the typical characteristics of Glässer's disease, including arthritis, fibrinous polyserositis, haemorrhagic pneumonia, and meningitis [24]. The SH0165 isolate was cultured at $37{ }^{\circ} \mathrm{C}$ for $12 \mathrm{~h}$ in tryptic soy broth (Difco Laboratories, USA) or grown for $24 \mathrm{~h}$ in tryptic soy agar (Difco Laboratories) supplemented with $10 \mu \mathrm{g} / \mathrm{mL}$ nicotinamide adenine dinucleotide (Sigma, USA) and 10\% foetal bovine serum (Gibco, Australia).

\section{Drugs}

Baicalin was provided by the National Institutes for Food and Drug Control (Beijing, B110715-201318). Before use, baicalin was dissolved and diluted with RPMI-1640 medium (Gibco, USA).

Ethyl pyruvate (EP) and flunixin meglumine (FM) were purchased from Shanghai Macklin Biochemical Co., Ltd. and Guangdong WenS Dahuanong Biotechnology Co., Ltd., respectively.

\section{Animals and experimental design}

Fifty-six 30-day-old naturally farrowed early-weaned piglets (Duroc $\times$ Landrace $\times$ Large White) weighing $8-10 \mathrm{~kg}$ were purchased from Wuhan Wannianqing Animal Husbandry Co., Ltd. (Wuhan, China) for the in vivo experiments. The piglets used in this study were weaned on day 23. The piglets were negative for antibodies directed against G. parasuis when tested with INgezim Haemophilus 11.HPS.K.1 (INgezim, Spain).

The 56 piglets were randomly divided into seven groups of eight piglets each: the negative control group, infection group, EP group, FM group, treatment group 1, treatment group 2, and treatment group 3. Before G. parasuis challenge, the piglets in the EP group were injected intraperitoneally with EP at $40 \mathrm{mg} / \mathrm{kg}$ body weight (BW); the piglets in the FM group were injected intramuscularly with FM at $2 \mathrm{mg} / \mathrm{kg} \mathrm{BW}$; and the piglets in treatment group 1, treatment group 2, and treatment group 3 were injected intramuscularly with baicalin at 25,50 , and $100 \mathrm{mg} / \mathrm{kg} \mathrm{BW}$, respectively. After $30 \mathrm{~min}$, the infection group, EP group, FM group, treatment group 1, treatment group 2, and treatment group 3 were challenged intraperitoneally with $1 \times 10^{9} \mathrm{CFU}$ of the G. parasuis strain in $2 \mathrm{~mL}$ of normal saline. The piglets from the negative control group were injected intraperitoneally with an equivalent volume of saline. The piglets in all the groups were monitored for 7 days after challenge, and their morbidity and mortality were recorded. 
Analysis of cytokine secretion and HMGB1 with enzyme-linked immunosorbent assay (ELISA) and real-time quantitative PCR (qPCR)

At $48 \mathrm{~h}$ following G. parasuis challenge, blood samples were collected for cytokine analysis. The presence of interleukin (IL) 1 $\beta$, IL6, IL8, IL10, tumour necrosis factor $\alpha$ (TNF- $\alpha$ ), and HMGB1 in the sera was detected with ELISA kits (for IL1 $\beta$, IL8, IL10, and HMGB1, Blue Gene, Shanghai, China; for IL6 and TNF- $\alpha$, $R \& D$ Systems, USA), according to the manufacturers' instructions. The expression of IL1 $\beta$, IL6, IL-8, IL10, TNF- $\alpha$, and HMGB1 mRNAs was also measured at the same time with qPCR. Peripheral blood monocytes (PBMNs) were isolated with a method previously established in our laboratory [19]. Total RNA was extracted from the PBMNs with TRIzol Reagent (Invitrogen, USA) and reverse transcribed into cDNA with reverse transcriptase (TaKaRa, China) and a SYBR Green PCR Kit (TaKaRa, China), according to the manufacturer's protocols. The transcription analysis of each sample was repeated at least three times. In this study, $\beta$-actin was used as the internal control. The primers used for the qPCRs are shown in Table 1.

\section{Determination of the effect of baicalin on the MAPK} signalling pathway and apoptosis by western blotting

The effects of baicalin on the MAPK signalling pathway and on apoptosis were determined as previously described, with some minor modifications [18]. Briefly, the aortic vessels of the piglets were collected, and the total protein was extracted with a total protein extraction kit (Beyotime Biotechnology, China). The protein concentrations were measured with a BCA protein assay kit (Sigma, USA). The isolated total proteins were resolved with $12 \%$ SDS-PAGE and transferred onto polyvinylidene difluoride membranes, which were then blocked with $5 \%$ skimmed milk for 90 min and washed five times with Tris-buffered saline containing Tween 20 (TBST). The membranes were incubated with the appropriate antibodies or anti- $\beta$-actin antibody (Cell Signaling Technology, USA) for $14 \mathrm{~h}$ at $4{ }^{\circ} \mathrm{C}$. After the membranes were washed five times with TBST, they were incubated with horseradish-peroxidase-linked goat anti-rabbit antibody (Proteintech, USA) for $30 \mathrm{~min}$ at $25{ }^{\circ} \mathrm{C}$ and visualized with ECL solution (Thermo Pierce ECL, USA). The protein expression levels of c-Jun N-terminal kinase (JNK), phosphorylated JNK (p-JNK), protein 38 (P38), p-P38, extracellular regulated protein kinases (ERK), p-ERK, and caspase 3 were determined with the FluorChem ${ }^{\mathrm{TM}}$ FC2 AIC system (Alpha Innotech, USA).
Table 1 Primer sequences for qRT-PCR

\begin{tabular}{|c|c|c|c|}
\hline Gene & Nucleotide Sequence $\left(5^{\prime}-3^{\prime}\right)$ & $\operatorname{Tm}\left({ }^{\circ} \mathrm{C}\right)$ & Length (bp) \\
\hline \multicolumn{4}{|l|}{$\beta$-actin } \\
\hline Forward & TGCGGGACATCAAGGAGAAG & 57.4 & 216 \\
\hline Reverse & AGTTGAAGGTGGTCTCGTGG & 57.4 & \\
\hline \multicolumn{4}{|l|}{$\mid L-1 \beta$} \\
\hline Forward & TCTGCATGAGCTTTGTGCAAG & 57.7 & 155 \\
\hline Reverse & ACAGGGCAGACTCGAATTCAAC & 55.8 & \\
\hline \multicolumn{4}{|l|}{ IL-6 } \\
\hline Forward & TGTCGAGGCTGTGCAGATTAGT & 57.7 & 142 \\
\hline Reverse & CATCCATCGTTCTGTGACTGC & 57.6 & \\
\hline \multicolumn{4}{|l|}{$\mid \mathrm{L}-8$} \\
\hline Forward & ACAGCAGTAACAACAACAAG & 50.1 & 117 \\
\hline Reverse & GACCAGCACAGGAATGAG & 50.2 & \\
\hline \multicolumn{4}{|l|}{ IL-10 } \\
\hline Forward & CGTGGAGGAGGTGAAGAGTG & 55.4 & 178 \\
\hline Reverse & TTAGTAGAGTCGTCATCCTGGAAG & 55.6 & \\
\hline \multicolumn{4}{|l|}{ TNF-a } \\
\hline Forward & CGCTCTTCTGCCTACTGCACTTC & 61.3 & 164 \\
\hline Reverse & CTGTCCCTCGGCTTTGACATT & 57.6 & \\
\hline \multicolumn{4}{|l|}{ HMGB1 } \\
\hline Forward & GATCCTAAGAAGCCGAGAG & 55.2 & 102 \\
\hline Reverse & GAAGTTGACTGAAGCATCTG & 53.4 & \\
\hline
\end{tabular}

\section{Histopathology}

Seven days after the G. parasuis SH0165 challenge, the brains and lungs of the surviving piglets from all groups were isolated, fixed in 10\% neutral-buffered formalin, and embedded in paraffin. Tissue sections $(4 \mu \mathrm{m}$ thick) were stained with haematoxylin and eosin with the standard method and observed with light microscopy [25].

\section{Statistical analysis}

The experimental data are shown as the means \pm SDs. Differences between two groups were analysed with Student's two-tailed $t$ test. Survival analysis was performed with a log rank test. $p$ values $<0.05$ were considered significant; $* 00.05$ and ${ }^{* * *} p<0.01$.

\section{Results}

Baicalin could protect piglets against $G$. parasuis challenge Baicalin provided $100 \%, 100 \%$, and $87.5 \%$ protection to treatment group 1, treatment group 2, and treatment group 3, respectively, compared with that for the infection group, which was non-significant $(p>0.05$, Figure 1). The mortality of the piglets in the infection group was $37.5 \%$ in the days after their challenge with G. parasuis strain SH0165, and treatment groups 1, 2, 


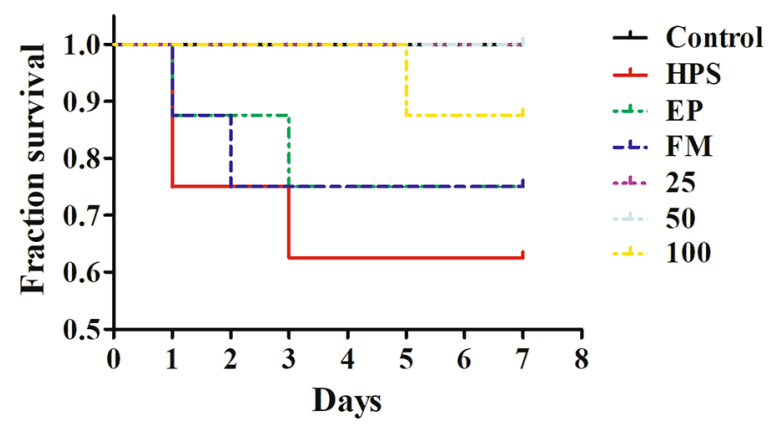

Figure 1 Effects of baicalin on the protection of piglets against G. parasuis challenge. Before $G$. parasuis challenge, the piglets in the EP group were injected intraperitoneally with EP, and the piglets in the FM group were injected intramuscularly with FM. After pretreatment with baicalin at concentrations of $25-100 \mathrm{mg} /$ $\mathrm{kg}$ BW for $30 \mathrm{~min}$, the piglets were challenged with $1 \times 10^{9} \mathrm{CFU}$ of $\mathrm{G}$. parasuis strain SH0165. The mortality of the piglets was recorded. G. parasuis: HPS (the infection group); $25-100$ : the piglets were injected intramuscularly with baicalin at $25 \mathrm{mg} / \mathrm{kg} \mathrm{BW}$ (treatment group 1), $50 \mathrm{mg} / \mathrm{kg}$ BW (treatment group 2), or $100 \mathrm{mg} / \mathrm{kg} \mathrm{BW}$ (treatment group 3).

and 3 were protected during the observation period (Figure 1).

\section{Effects of baicalin on the release of cytokines in the serum of piglets challenged with $\mathrm{G}$. parasuis}

The levels of IL1 $\beta$, IL6, IL8, IL10, and TNF- $\alpha$ were significantly higher after $G$. parasuis challenge than in the control condition, as measured by ELISA $(p<0.01$, Figure 2A). EP did not significantly reduce IL6, IL10, and TNF- $\alpha$ secretion ( $p>0.05$, Figure 2A), but EP significantly inhibited the production of IL1 $\beta$ and IL8 $(p<0.01$, Figure 2A). FM did not significantly reduce IL1 $\beta$ and IL10 release ( $p>0.05$, Figure $2 \mathrm{~A})$, but FM significantly attenuated the production of IL6, IL8, and TNF- $\alpha$, as measured by ELISA $(p<0.05$ for IL6, and $p<0.01$ for IL8 and TNF- $\alpha$, Figure 2A). Baicalin at concentrations of $50-100 \mathrm{mg} / \mathrm{kg}$ BW reduced the secretion of IL6, IL8, and TNF- $\alpha$, as measured by ELISA $(p<0.05$ for TNF- $\alpha$ and $p<0.01$ for IL6 and IL8, Figure 2A). G. parasuis also increased the expression of IL1 $\beta$, IL6, IL8, IL10, and TNF- $\alpha$ mRNAs relative to that in the control condition $(p<0.01$, Figure $2 \mathrm{~B})$. EP significantly attenuated the expression of IL1 $\beta$, IL8, and IL10 $(p<0.01$, Figure 2B). FM significantly reduced the expression of IL1 $\beta$, IL6, IL8, IL10, and TNF- $\alpha(p<0.05$ for TNF- $\alpha$, $p<0.01$ for IL1 $\beta$, IL6, IL8, and IL10, Figure 2B). Baicalin at concentrations of $25-50 \mathrm{mg} / \mathrm{kg} \mathrm{BW}$ reduced the expression of IL1 $\beta$, IL6, IL8, IL10, and TNF- $\alpha(p<0.05$ for TNF- $\alpha, p<0.01$ for IL1 $\beta$, IL6, IL8, and IL10, Figure 2B).
Effects of baicalin on the release of HMGB1 into the serum of piglets challenged with G. parasuis

After the piglets were challenged with G. parasuis, the levels of HMGB1 were significantly higher in the non-treated group than in the non-infected group, as measured by ELISA $(p<0.01$, Figure $3 \mathrm{~A})$. EP and FM reduced the release of HMGB1 $(p<0.01$, Figure $3 A)$, and $25-100 \mathrm{mg} / \mathrm{kg}$ baicalin significantly reduced its release, according to ELISA results $(p<0.05$ for $50, p<0.01$ for 25 and $100 \mathrm{mg} / \mathrm{kg}$, Figure 3A). HMGB1 expression at the mRNA level was consistent with the ELISA results $(p<0.01$, Figure 3B).

\section{Effects of baicalin on cell apoptosis induced by G. parasuis}

To explore the effects of baicalin on the apoptosis induced by G. parasuis, the expression of caspase 3 was measured by western blotting. The results demonstrated that $G$. parasuis increased cell apoptosis after the piglets were challenged with the bacterium compared with the level in the control piglets $(p<0.01$, Figure 4$)$, but caspase 3 expression was inhibited in the EP and FM groups $(p<0.05$, Figure 4). Baicalin $(25-100 \mathrm{mg} / \mathrm{kg} \mathrm{BW})$ also attenuated cell apoptosis in piglets challenged with $G$. parasuis compared with that in the infection group piglets $(p<0.05$ for $25, p<0.01$ for 50 and 100, Figure 4).

\section{Effects of baicalin on the MAPK signalling pathway activated by $G$. parasuis}

The data showed that G. parasuis promoted the phosphorylation of ERK, JNK, and P38 compared with that in the control condition $(p<0.05$ for ERK and JNK, $p<0.01$ for P38, Figure 5B, D, F), whereas the phosphorylation of ERK, JNK, and P38 in piglets challenged with G. parasuis was inhibited by FM $(p<0.05$, Figure $5 \mathrm{~B}, \mathrm{D}, \mathrm{F})$, and EP attenuated the phosphorylation of P38 in these piglets $(p<0.01$, Figure $5 \mathrm{~F})$. Baicalin $(50-100 \mathrm{mg} / \mathrm{kg} \mathrm{BW})$ suppressed ERK, JNK and P38 phosphorylation in piglets infected with G. parasuis $(p<0.01$, Figure $5 B, D, F)$.

\section{Histopathological analysis}

The piglets from the G. parasuis infection group displayed severe pathological damage to their lung and brain tissues (Figures 6-HPS, 7-HPS). However, only mild tissue damage was detected in the surviving piglets of the EP, FM, and baicalin treatment groups (Figures 6 and 7). In the G. parasuis infection group, extensive proliferation of fibroblasts was detected in the lung parenchyma and massive neutrophil infiltration in the bronchioles (Figure 6-HPS). Inflammatory cell infiltration and aggregation were detected in the brains of the G. parasuis infection group (Figure 7HPS). However, only minor histopathological damage was detected in the lungs or brains of the piglets in the EP, FM, 


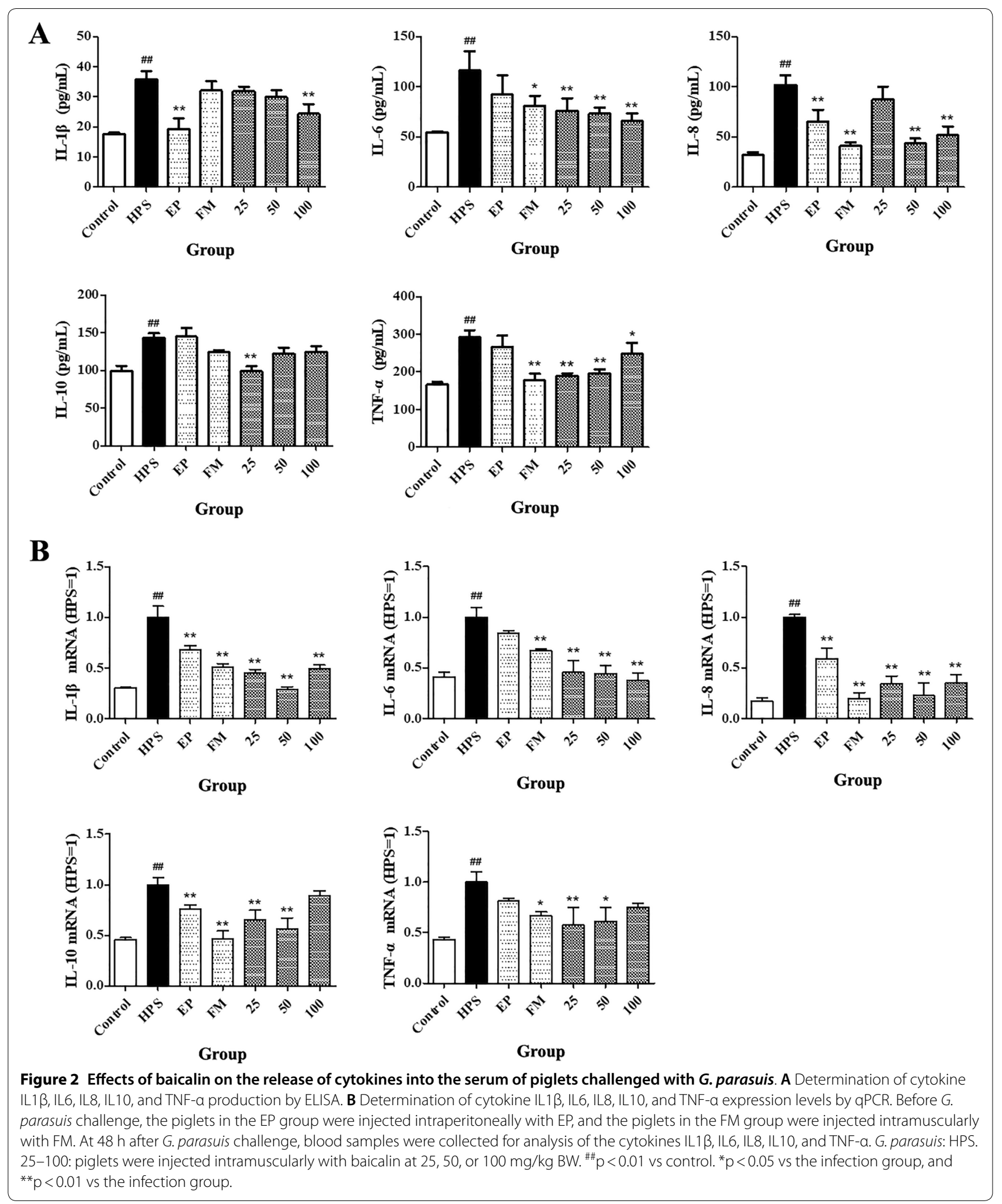




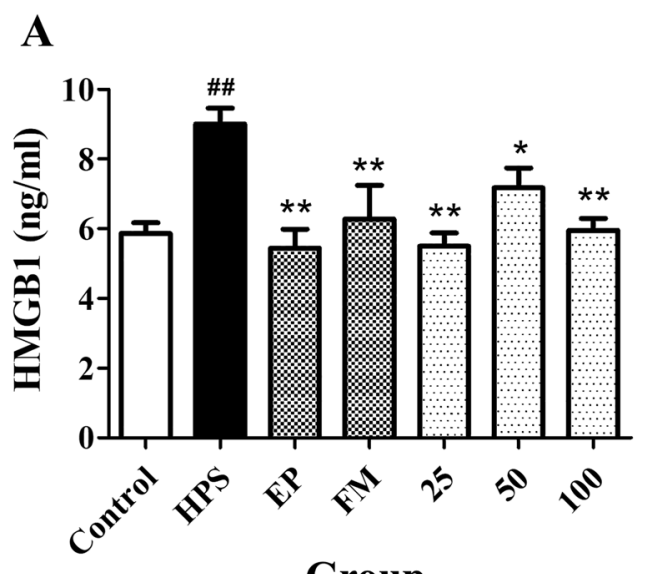

Group

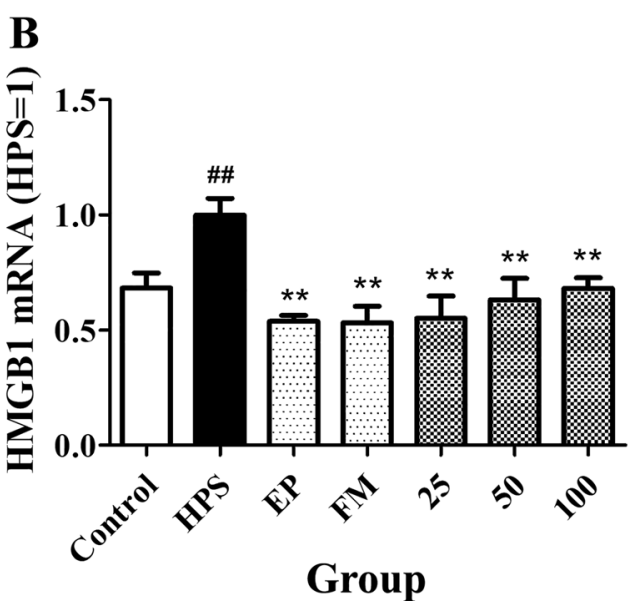

Group

Figure 3 Effects of baicalin on the release of HMGB1 into the serum of piglets challenged with G. parasuis. A Determination of HMGB1 production by ELISA. B Determination of the HMGB1 expression level by qPCR. Before G. parasuis challenge, the piglets in the EP group were injected intraperitoneally with EP, and the piglets in the FM group were injected intramuscularly with FM. At $48 \mathrm{~h}$ after G. parasuis challenge, blood samples were collected to measure the HMGB1 levels. G. parasuis: HPS. 25-100: the piglets were injected intramuscularly with baicalin at 25, 50, or $100 \mathrm{mg} / \mathrm{kg} \mathrm{BW.}{ }^{\# \#} \mathrm{p}<0.01$ vs control. ${ }^{*} p<0.05$ vs the infection group, and ${ }^{* *} p<0.01$ vs the infection group.

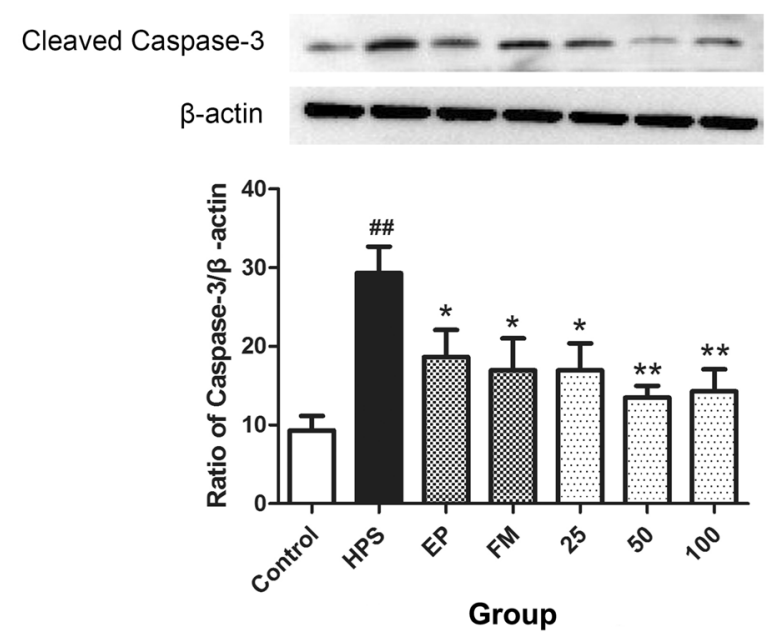

Figure 4 Effects of baicalin on cell apoptosis induced by $G$. parasuis. Before G. parasuis challenge, the piglets in the EP group were injected intraperitoneally with EP, and the piglets in the FM group were injected intramuscularly with FM. Aortic vessels were collected, and the expression of caspase 3 was measured by western blotting. G. parasuis: HPS. 25-100: the piglets were injected intramuscularly with baicalin at 25,50 , or $100 \mathrm{mg} / \mathrm{kg} \mathrm{BW.}{ }^{\#} \mathrm{p}<0.01$ vs control. ${ }^{*} p<0.05$ vs the infection group and ${ }^{* *} p<0.01$ vs the infection group. and baicalin treatment groups (Figure 6-EP, FM, 25, 50, 100; Figure 7-EP, FM, 25, 50, 100).

\section{Discussion}

In this study, we comprehensively investigated the antiinflammatory efficacy of baicalin in piglets challenged with G. parasuis and the mechanism of its anti-inflammatory action. Our results demonstrate that baicalin could provide certain protection to piglets against G. parasuis challenge but not significantly, which might be related to the numbers of piglets. In the future, we will use more animals to study the protection of piglets against G. parasuis challenge afforded by baicalin. We also found that the anti-inflammatory effect of baicalin may involve the attenuation of cell apoptosis, the reduction in HMGB1 release, and the inhibition of the activation of the MAPK signalling pathway.

In this study, EP and FM were used as control drugs. Previous research has reported that EP reduced renal damage during renal diseases induced with methylglyoxal-derived advanced glycation end products [26]. EP reduced the release of IL8, TNF- $\alpha$, IL6, and IL1 $\beta$ and attenuated apoptosis in LPS-induced inflammation in IPEC-J2 cells [27]. It effectively inhibited HMGB1 and receptor for advanced glycation end products (RAGE) expression, thus reducing

(See figure on next page.)

Figure 5 Effects of baicalin on the MAPK signalling pathway activated by G. parasuis. Before G. parasuis challenge, the piglets in the EP group were injected intraperitoneally with EP, and the piglets in the FM group were injected intramuscularly with FM. Aortic vessels were collected, and the protein expression levels of ERK (A), p-ERK (B), JNK (C), p-JNK (D), P38 (E) and p-P38 (F) were determined. G. parasuis: HPS. 25-100: the piglets were injected intramuscularly with baicalin at 25,50 , or $100 \mathrm{mg} / \mathrm{kg} \mathrm{BW.}{ }^{\# \#} p<0.01$ vs control. ${ }^{*} p<0.05$ vs the infection group, and ${ }^{* *} p<0.01$ vs the infection group. 

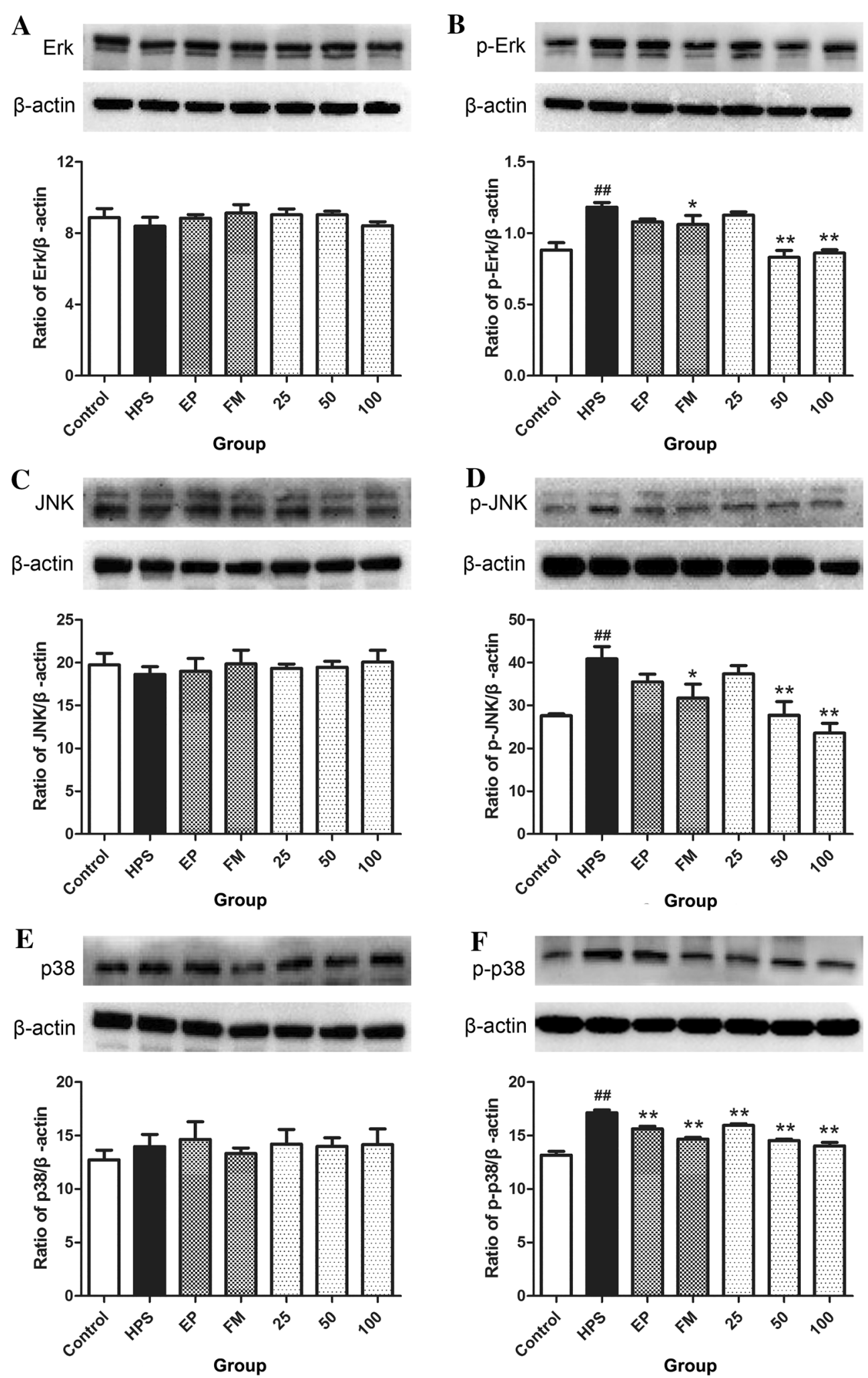


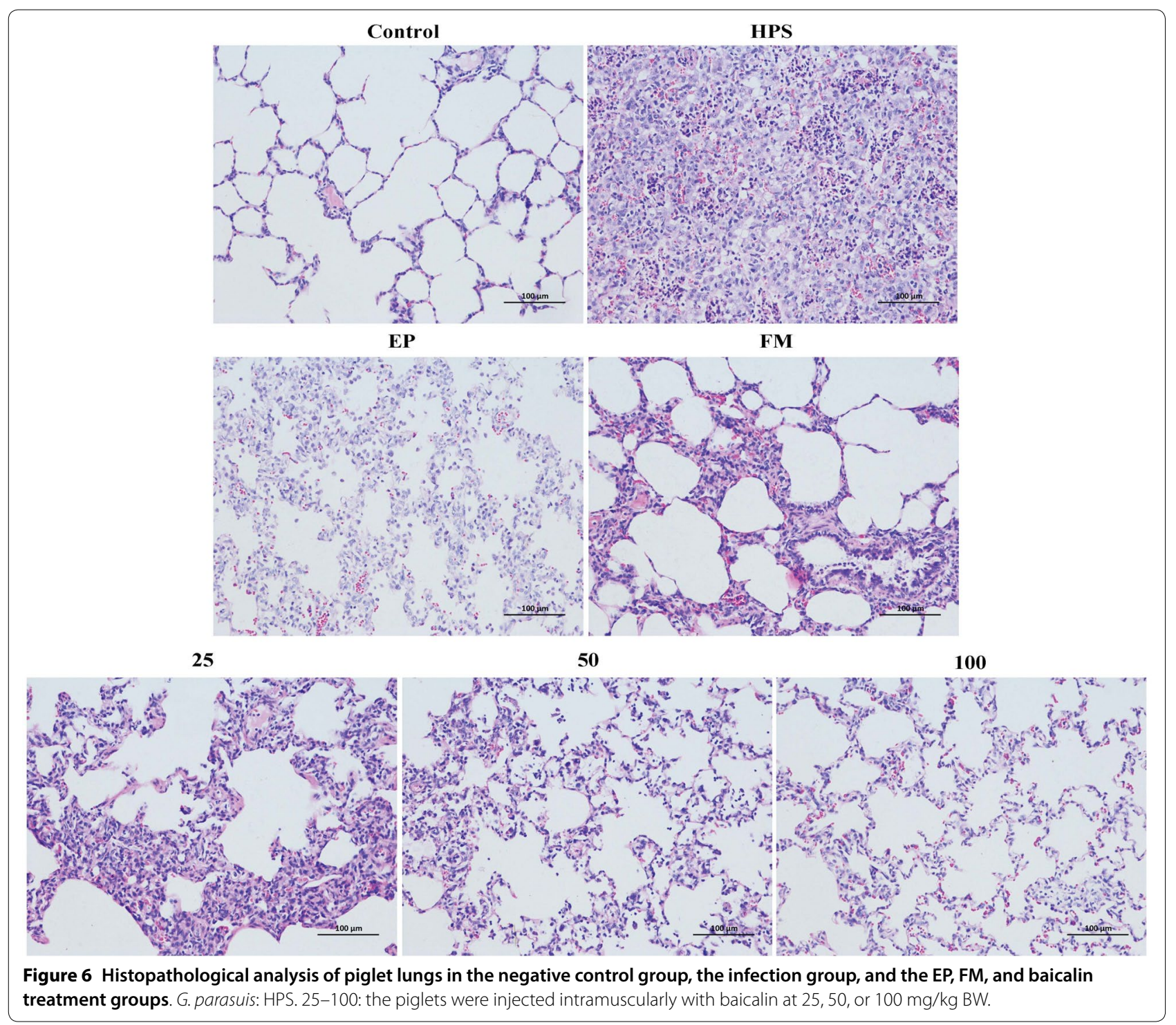

the activity of the HMGB1-RAGE axis, and is therefore considered an inhibitor of HMGB1 [28]. Consistent with previous results, our data also showed that EP attenuates the expression of HMGB1 in piglets infected with G. parasuis. It has been documented that FM exerts significant antiinflammatory effects in piglets and cattle [29,30]. Flunixin meglumine also reduces the release of the cytokines IL1 $\beta$, IL10, and TNF- $\alpha$ [31], so it is considered a good option for controlling inflammation-related diseases, such as G. parasuis infection.

Cytokines are important mediators of inflammation, immunity, and the pathological damage that occurs during the disease processes [32]. Previous research reported that IL1 $\beta$, IL6, and IL8 were produced when pigs were challenged with G. parasuis, which could be considered one of the characteristics of G. parasuis infection [33, 34]. The expression levels of IL-10 and TNF- $\alpha$ in the spleen were upregulated after colostrum-deprived pigs were infected with G. parasuis [35]. Stimulation of Raw 264.7 macrophages with G. parasuis PotD could induce the production of IL1 $\beta$, IL6, and TNF- $\alpha$ [36]. Therefore, these cytokines play important roles in the inflammatory damage caused by G. parasuis infection. Consequently, we quantified the expression of these cytokines (IL1 $\beta$, IL6, IL8, IL10, and TNF- $\alpha$ ) at both the mRNA and protein levels. Our results confirmed that G. parasuis induced IL1 $\beta$, IL6, IL8, IL10, and TNF- $\alpha$ expression, and baicalin attenuated the production of all these cytokines in piglets challenged with G. parasuis, confirming that baicalin has anti-inflammatory activity. However, the 


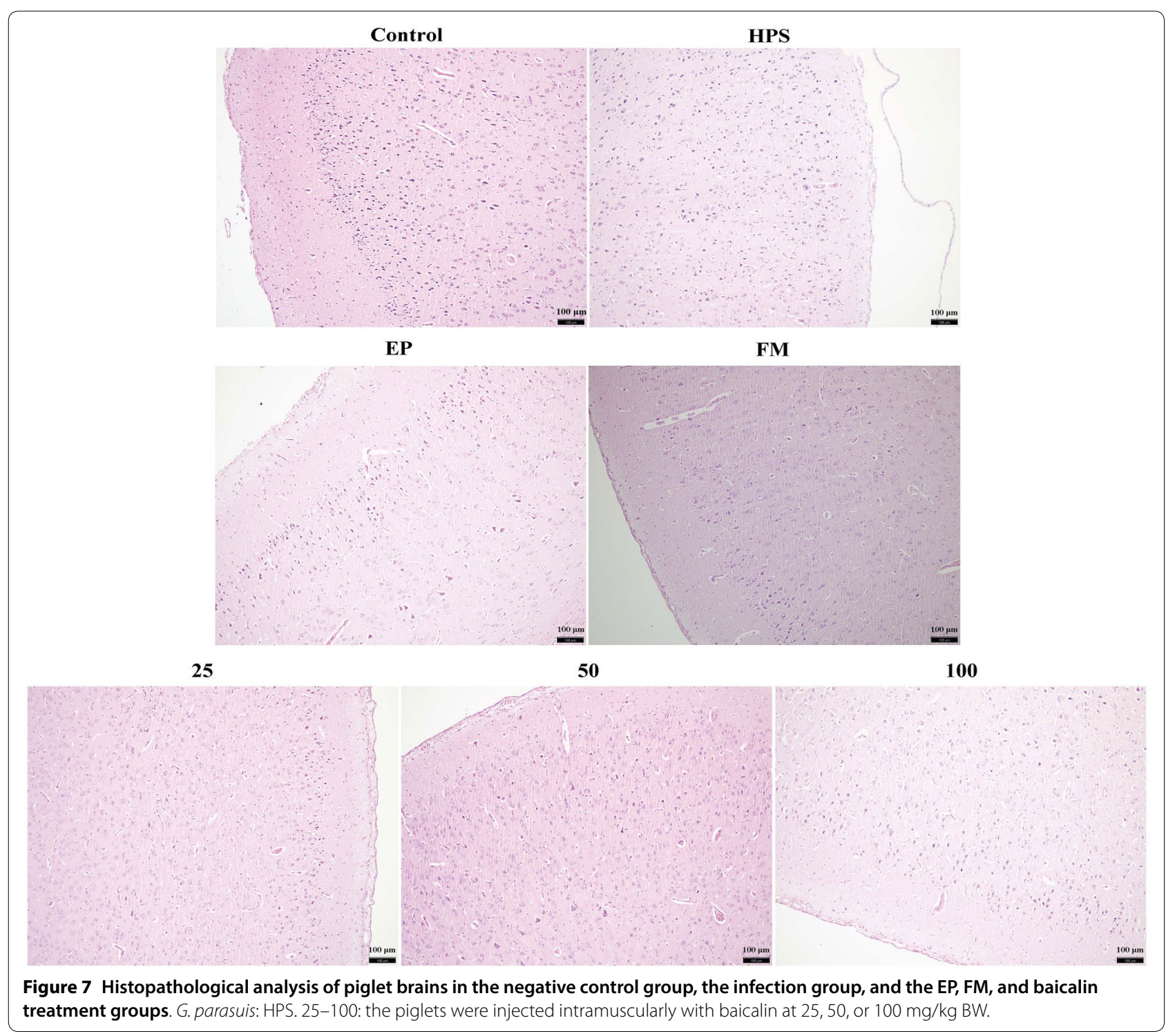

special characteristics of these cytokines in the inflammatory damage that occurs during the pathogenesis of $G$. parasuis infection require further research.

HMGB1 is a ubiquitous nuclear protein that regulates the expression of key genes involved in the pathogenesis of many diseases [37]. Previous research has shown that HMGB1 released from mouse trophoblasts contributes to inflammation during Brucella melitensis infection [38]. HMGB1 is reported to induce signalling via the RAGE receptor and the HMGB1/RAGE/cathepsin B signalling pathway, which activates NLRP3-dependent coronary endothelial cell pyroptosis and thus plays an important role in the endothelial damage that occurs in Kawasaki disease [39]. The HMGB1-RAGE proinflammatory axis promotes vascular inflammation and endothelial cell apoptosis, resulting in vascular injury during acute limb ischaemia and reperfusion [40]. HMGB1 is also involved in acute lung injury triggered in mice by LPS by activating the RAGE/NF- $\mathrm{KB}$, tolllike receptor 2 (TLR)2, and TLR4 signalling pathways [41]. HMGB1 also participates in systemic fibrotic diseases through the RAGE/MAPK and NF- $\mathrm{KB}$ signalling pathways [42]. In a previous study, we showed that $G$. parasuis stimulated the release of HMGB1 in piglet PBMNs [22] and induced the activation of the RAGE and MAPK signalling pathways in porcine aortic vascular endothelial cells [20]. Therefore, we investigated whether HMGB1 is released and the MAPK signalling pathway is activated in piglets after challenge with $G$. parasuis. Our results show that G. parasuis triggers 
the release of HMGB1 and the activation of the MAPK pathway in piglets and that baicalin significantly inhibits the production of HMGB1 and the activation of the MAPK pathway in these piglets. These data suggest that the HMGB1 and MAPK signalling pathways are involved in the inflammatory process or inflammatory damage during G. parasuis infection and could offer a novel therapeutic target to control Glässer's disease. In future studies, different specific inhibitors of the MAPK signalling pathway will be used to further investigate the mechanism of baicalin action.

Natural target animals are more suitable for examining the functions, metabolism, and efficacy of substances directed against the specific pathogen in vivo [43]. Therefore, the target animal of G. parasuis, the piglet, was selected to study the function of baicalin. Our results demonstrate that baicalin could provide certain protection to piglets against $G$. parasuis challenge and may have utility as a new natural antibiotic-free drug to treat $G$. parasuis infection.

Cell apoptosis is a type of programmed cell death that may contribute to the orderly and efficient removal of damaged cells [44]. In a previous study, we also showed that baicalin inhibits the apoptosis induced by G. parasuis via the RAGE and MAPK signalling pathways in vascular endothelial cells [20]. However, whether baicalin attenuates apoptosis in piglets challenged with $G$. parasuis has not been investigated until now. Our results demonstrate that baicalin reduces cell apoptosis in piglets challenged with $G$. parasuis.

In conclusion, our results show that baicalin could protect piglets against G. parasuis challenge, which may be related to the anti-inflammatory effect it exerts by inhibiting the activation of HMGB1, cell apoptosis, and the MAPK signalling pathway. Our data extend our knowledge of the pharmacological properties of baicalin, which may have utility as a novel therapeutic drug for the prevention of Glässer's disease.

\begin{abstract}
Abbreviations
G. parasuis: Glaesserella parasuis; IL: interleukin; TNF-a: tumour necrosis factor a; HMGB1: high mobility group box 1; MAPK: mitogen-activated protein kinase; PAM: porcine alveolar macrophage; LOS: lipooligosaccharide; NF-KB: nuclear factor KB; LPS: lipopolysaccharide; TUG1: taurine upregulated 1; APEC: avian pathogenic Escherichia coli; PAVECs: porcine aortic vascular endothelial cells; PMNPs: piglet mononuclear phagocytes; EP: ethyl pyruvate; FM: flunixin meglumine; BW: body weight; ELISA: enzyme-linked immunosorbent assay; qPCR: real-time quantitative PCR; PBMNs: peripheral blood monocytes; TBST: tris-buffered saline containing Tween 20; JNK: c-Jun N-terminal kinase; p-JNK: phosphorylated JNK; P38: protein 38; ERK: extracellular regulated protein kinase; RAGE: receptor for advanced glycation end products; TLR2: toll-like receptor 2 .
\end{abstract}

\section{Authors' contributions}

YQ and SF conceived and designed the experiments. SF, RY, SZ, JL, and YZ performed the experiments. YQ, LG, CY, YL, ZW, YH, and CH analysed the data. SF and $Y Q$ wrote the paper. All authors read and approved the final manuscript.

\section{Funding}

This work was supported by the National Natural Science Foundation of China (Grant No. 31572572)

\section{Availability of data and materials}

All data generated or analysed during this study are included in this published article.

\section{Ethics approval and consent to participate}

This study was performed in strict accordance with the recommendations of the China Regulations for the Administration of Affairs Concerning Experimental Animals 1988 and the Hubei Regulations for the Administration of Affairs Concerning Experimental Animals 2005. The protocol was approved by the China Hubei Province Science and Technology Department (permit number SYXK[ER] 2010-0029). All experimental animals were euthanized at the end of the experiment.

\section{Consent to publish}

Not applicable.

\section{Competing interests}

The authors declare that they have no competing interests.

\section{Author details}

${ }^{1}$ Hubei Key Laboratory of Animal Nutrition and Feed Science, Wuhan Polytechnic University, Wuhan 430023, People's Republic of China. ${ }^{2}$ Hubei Collaborative Innovation Center for Animal Nutrition and Feed Safety, Wuhan 430023, People's Republic of China. ${ }^{3}$ Biochemistry and Molecular Biology, University of New Mexico School of Medicine, Albuquerque, NM 87131, USA.

Received: 25 March 2020 Accepted: 4 August 2020

Published online: 14 August 2020

\section{References}

1. Oliveira S, Pijoan C (2004) Haemophilus parasuis: new trends on diagnosis, epidemiology and control. Vet Microbiol 99:1-12

2. Kielstein P, Rapp-Gabrielson VJ (1992) Designation of 15 serovars of Haemophilus parasuis on the basis of immunodiffusion using heat-stable antigen extracts. J Clin Microbiol 30:862-865

3. Cai X, Chen H, Blackall PJ, Yin Z, Wang L, Liu Z, Jin M (2005) Serological characterization of Haemophilus parasuis isolates from China. Vet Microbiol 111:231-236

4. Fu S, Zhang M, Xu J, Ou J, Wang Y, Liu H, Liu J, Chen H, Bei W (2013) Immunogenicity and protective efficacy of recombinant Haemophilus parasuis SH0165 putative outer membrane proteins. Vaccine 31:347-353

5. Zhou Y, Feng S, He X, Zhou Q, Wang Y, Yue H, Tang C, Zhang B (2019) Surface-exposed loops L7 and L8 of Haemophilus (Glaesserella) parasuis OmpP2 contribute to the expression of proinflammatory cytokines in porcine alveolar macrophages. Vet Res 50:105

6. Costa-Hurtado M, Garcia-Rodriguez L, Lopez-Serrano S, Aragon V (2019) Haemophilus parasuis VtaA2 is involved in adhesion to extracellular proteins. Vet Res 50:69

7. Shen Y, Zhou N, An J, Zhang J, Wang M, Li Y, Jiang P (2019) Haemophilus parasuis infection in 3D4/21 cells induces autophagy through the AMPK pathway. Cell Microbiol 21:13031

8. Zeng Z, Chen X, Yue H, He H, Ren Y, Tang C, Zhang B (2018) The effect of $\mathrm{rfaD}$ and $\mathrm{rfaF}$ of Haemophilus parasuis on lipooligosaccharide induced inflammation by NF-kB/MAPKs signaling in porcine alveolar macrophages. J Vet Med Sci 80:842-845

9. Zhang B, Ku X, Zhang X, Zhang Y, Chen G, Chen F, Zeng W, Li J, Zhu L, He Q (2019) The Al-2/luxS Quorum Sensing System Affects the Growth Characteristics, Biofilm Formation, and Virulence of Haemophilus parasuis. Front Cell Infect Microbiol 9:62

10. Hua K, Li Y, Zhou H, Hu X, Chen Y, He R, Luo R, Zhou R, Bi D, Jin H (2018) Haemophilus parasuis Infection Disrupts Adherens Junctions and Initializes EMT Dependent on Canonical Wnt/ $\beta$-Catenin Signaling Pathway. Front Cell Infect Microbiol 8:324 
11. He L, Dai K, Wen X, Ding L, Cao S, Huang X, Wu R, Zhao Q, Huang Y, Yan $Q$, Ma X, Han X, Wen Y (2018) QseC mediates osmotic stress resistance and biofilm formation in Haemophilus parasuis. Front Microbiol 9:212

12. Zhang B, Tang C, Liao M, Yue H (2014) Update on the pathogenesis of Haemophilus parasuis infection and virulence factors. Vet Microbiol 168:1-7

13. Huang Y, Sun M, Yang X, Ma A, Ma Y, Zhao A (2019) Baicalin relieves inflammation stimulated by lipopolysaccharide via upregulating TUG1 in liver cells. J Physiol Biochem 75:463-473

14. Wang WW, Jia HJ, Zhang HJ, Wang J, Lv HY, Wu SG, Qi GH (2019) Supplemental plant extracts from flos lonicerae in combination with baikal skullcap attenuate intestinal disruption and modulate gut microbiota in laying hens challenged by Salmonella pullorum. Front Microbiol 10:1681

15. Peng LY, Yuan M, Wu ZM, Song K, Zhang $C L$, An Q, Xia F, Yu JL, Yi PF, Fu BD, Shen HQ (2019) Anti-bacterial activity of baicalin against APEC through inhibition of quorum sensing and inflammatory responses. Sci Rep 9:4063

16. Chen ME, Su CH, Yang JS, Lu CC, Hou YC, Wu JB, Hsu YM (2018) Baicalin, Baicalein, and Lactobacillus Rhamnosus JB3 Alleviated Helicobacter pylori Infections in Vitro and in Vivo. J Food Sci 83:3118-3125

17. Wu SC, Chu XL, Su JQ, Cui ZQ, Zhang LY, Yu ZJ, Wu ZM, Cai ML, Li HX, Zhang ZJ (2018) Baicalin protects mice against Salmonella typhimurium infection via the modulation of both bacterial virulence and host response. Phytomedicine 48:21-31

18. Fu S, Liu H, Xu L, Qiu Y, Liu Y, Wu Z, Ye C, Hou Y, Hu CA (2018) Baicalin modulates NF-KB and NLRP3 inflammasome signaling in porcine aortic vascular endothelial cells Infected by Haemophilus parasuis Causing Glässer's disease. Sci Rep 8:807

19. Fu S, Xu L, Li S, Qiu Y, Liu Y, Wu Z, Ye C, Hou Y, Hu CA (2016) Baicalin suppresses NLRP3 inflammasome and nuclear factor-kappa B (NF-KB) signaling during Haemophilus parasuis infection. Vet Res 47:80

20. Fu S, Zhao W, Xiong C, Guo L, Guo J, Qiu Y, Hu CA, Ye C, Liu Y, Wu Z, Hou Y (2019) Baicalin modulates apoptosis via RAGE, MAPK, and AP-1 in vascular endothelial cells during Haemophilus parasuis invasion. Innate Immun 25:420-432

21. Ye C, Li R, Xu L, Qiu Y, Fu S, Liu Y, Wu Z, Hou Y, Hu CA (2019) Effects of Baicalin on piglet monocytes involving PKC-MAPK signaling pathways induced by Haemophilus parasuis. BMC Vet Res 15:98

22. Fu S, Liu H, Chen X, Qiu Y, Ye C, Liu Y, Wu Z, Guo L, Hou Y, Hu CA (2018) Baicalin Inhibits Haemophilus Parasuis-Induced High-Mobility Group Box 1 Release during Inflammation. Int J Mol Sci 19:1307

23. Guo L, Liu J, Zhang Y, Fu S, Qiu Y, Ye C, Liu Y, Wu Z, Hou Y, Hu CA (2020) The effect of baicalin on the expression profiles of long non-coding RNAs and mRNAs in porcine aortic vascular endothelial cells infected with Haemophilus parasuis. DNA Cell Biol 39:801-815

24. Yue M, Yang F, Yang J, Bei W, Cai X, Chen L, Dong J, Zhou R, Jin M, Jin Q, Chen $\mathrm{H}$ (2009) Complete genome sequence of Haemophilus parasuis SH0165. J Bacteriol 191:1359-1360

25. Singh H, Arora G, Nayak B, Sharma A, Singh G, Kumari K, Jana S, Patel C, Pandey AK, Seth A, Kumar R (2020) Semi-quantitative F-18-FDG PET/ computed tomography parameters for prediction of grade in patients with renal cell carcinoma and the incremental value of diuretics. Nucl Med Commun 41:485-493

26. Jung E, Kang WS, Jo K, Kim J (2019) Ethyl pyruvate prevents renal damage induced by methylglyoxal-derived advanced glycation end products. J Diabetes Res 2019:4058280

27. Dong N, Xu X, Xue C, Wang C, Li X, Bi C, Shan A (2019) Ethyl pyruvate inhibits LPS induced IPEC-J2 inflammation and apoptosis through p38 and ERK1/2 pathways. Cell Cycle 18:2614-2628

28. Liu Q, Huo Y, Zheng H, Zhao J, Jia L, Wang P (2019) Ethyl pyruvate suppresses the growth, invasion and migration and induces the apoptosis of non small cell lung cancer cells via the HMGB1/RAGE axis and the NF-KB/ STAT3 pathway. Oncol Rep 42:817-825

29. Levionnois OL, Fosse TK, Ranheim B (2018) PK/PD modeling of flunixin meglumine in a kaolin-induced inflammation model in piglets. J Vet Pharmacol Ther 41:314-323
30. Thiry J, Fournier R, Roy O, Catala M (2017) Evaluation of flunixin meglumine pour-on administration on prostaglandin E2 concentration in inflammatory exudate after induction of inflammation in cattle. Res Vet Sci 114:294-296

31. Yazar E, Er A, Uney K, Altunok V, Elmas M (2007) Effect of flunixin meglumine on cytokine levels in experimental endotoxemia in mice. J Vet Med A Physiol Pathol Clin Med 54:352-355

32. Borthwick LA (2016) The IL-1 cytokine family and its role in inflammation and fibrosis in the lung. Semin Immunopathol 38:517-534

33. Frandoloso R, Martínez-Martínez S, Rodríguez-Ferri EF, Yubero S, Rodríguez-Lázaro D, Hernández M, Gutiérrez-Martín CB (2013) Haemophilus parasuis subunit vaccines based on native proteins with affinity to porcine transferrin prevent the expression of proinflammatory chemokines and cytokines in pigs. Clin Dev Immunol 2013:132432

34. Costa-Hurtado M, Olvera A, Martinez-Moliner V, Galofré-Milà N, Martínez P, Dominguez J, Aragon V (2013) Changes in macrophage phenotype after infection of pigs with Haemophilus parasuis strains with different levels of virulence. Infect Immun 81:2327-2333

35. de la Fuente AJ, Ferri EF, Tejerina F, Frandoloso R, Martínez SM, Martín CB (2009) Cytokine expression in colostrum-deprived pigs immunized and challenged with Haemophilus parasuis. Res Vet Sci 87:47-52

36. Dai $K$, Ma X, Yang Z, Chang YF, Cao S, Zhao Q, Huang X, Wu R, Huang Y, Yan Q, Han X, Ma X, Wen X, Wen Y (2019) Polyamine transport protein PotD protects mice against Haemophilus parasuis and elevates the secretion of pro-inflammatory cytokines of macrophage via JNK-MAPK and NF-KB signal pathways through TLR4. Vaccines 7:216

37. Wang M, Gauthier A, Daley L, Dial K, Wu J, Woo J, Lin M, Ashby C, Mantell LL (2019) The role of HMGB1, a nuclear damage-associated molecular pattern molecule, in the pathogenesis of lung diseases. Antioxid Redox Signal 31:954-993

38. Liu X, Zhou M, Wu J, Wang J, Peng Q (2019) HMGB1 release from trophoblasts contributes to inflammation during Brucella melitensis infection. Cell Microbiol 21:13080

39. Jia C, Zhang J, Chen H, Zhuge Y, Chen H, Qian F, Zhou K, Niu C, Wang F, Qiu H, Wang Z, Xiao J, Rong X, Chu M (2019) Endothelial cell pyroptosis plays an important role in Kawasaki disease via HMGB1/RAGE/cathespin B signaling pathway and NLRP3 inflammasome activation. Cell Death Dis 10:778

40. Mi L, Zhang $Y, X u Y$, Zheng $X$, Zhang $X$, Wang Z, Xue M, Jin X (2019) HMGB1/RAGE pro-inflammatory axis promotes vascular endothelial cell apoptosis in limb ischemia/reperfusion injury. Biomed Pharmacother 116:109005

41. Wang J, Li R, Peng Z, Hu B, Rao X, Li J (2020) HMGB1 participates in LPS-induced acute lung injury by activating the AIM2 inflammasome in macrophages and inducing polarization of $\mathrm{M} 1$ macrophages via TLR2, TLR4, and RAGE/NF-KB signaling pathways. Int J Mol Med 45:61-80

42. Kim J, Park JC, Lee MH, Yang CE, Lee JH, Lee WJ (2017) High-mobility group box 1 mediates fibroblast activity via RAGE-MAPK and NF-KB signaling in keloid scar formation. Int J Mol Sci 19:76

43. Marra A (2020) Animal models for drug development for MRSA. Methods Mol Biol 2069:253-266

44. Pistritto G, Trisciuoglio D, Ceci C, Garufi A, D'Orazi G (2016) Apoptosis as anticancer mechanism: function and dysfunction of its modulators and targeted therapeutic strategies. Aging 8:603-619

\section{Publisher's Note}

Springer Nature remains neutral with regard to jurisdictional claims in published maps and institutional affiliations. 